RECENT DEVELOPMENTS IN THE RESPONSE MATRIX METHOD

by

R. J. Pryor

Savannah River Laboratory

E. I. du Pont de Nemours and Company

Aiken, South Carolina 29801

An invited paper for presentation at a meeting sponsored by the American Nuclear Society entitled "Advanced Reactors; Physics, Design, and Economics" to be held in Atlanta, Georgia, on September 8-11, 1974.

This paper was prepared in connection with work under Contract No. AT $(07-2)-1$ with the U. S. Atomic Energy Commission. By acceptance of this paper, the publisher and/or recipient acknowledges the U. S. Government's right to retain a non-exclusive, royalty-free license in and to any copyright covering this paper, along with the right to reproduce and to authorize others to reproduce all or any part of the copyrighted paper. 


\section{DISCLAIMER}

This report was prepared as an account of work sponsored by an agency of the United States Government. Neither the United States Government nor any agency Thereof, nor any of their employees, makes any warranty, express or implied, or assumes any legal liability or responsibility for the accuracy, completeness, or usefulness of any information, apparatus, product, or process disclosed, or represents that its use would not infringe privately owned rights. Reference herein to any specific commercial product, process, or service by trade name, trademark, manufacturer, or otherwise does not necessarily constitute or imply its endorsement, recommendation, or favoring by the United States Government or any agency thereof. The views and opinions of authors expressed herein do not necessarily state or reflect those of the United States Government or any agency thereof. 


\section{DISCLAIMER}

Portions of this document may be illegible in electronic image products. Images are produced from the best available original document. 


\title{
RECENT DEVELOPMENTS IN THE RESPONSE MATRIX METHOD*
}

\author{
R. J. Pryor \\ Savannah River Laboratory \\ E. I. du Pont de Nemours and Company \\ Aiken, South Carolina 29801
}

\begin{abstract}
Recent developments in the response matrix method are discussed. Particular attention is given to the derivation of the static response matrix, and two alternative approaches to the time-dependent problem are consldered. Solution methods and future developments are also discussed.
\end{abstract}

\section{INTRODUCTION}

The response matrix method has gained in popularity recently partly because such calculations are becoming economically feastble but more importantly because the analysis of today's reactors demands a more exact treatment than afforded by diffusion theory. The. response matrix method offers its users the ability to treat the entire reactor (with as much precision as desired), providing a full transport solution of the reactor flux with the heterogeneous core components treated in detall. As a result, no secondary homogeneous calculations are required, and both local and global nuclear effects are treated simultaneously. This permits accurate prediction of key reactor parameters such as control rod worth and reactivity. The response matrix method is capable of treating most reactor design concepts and has been successfully applied for two-dimensional static problems to heavy water and light water reactors and to the LMFBR $(\underline{1,2})$. Recent work has extended this method to treat translent problems ( 3 ).

The response matrix method can be viewed as a multidimensional extension of the invariant embedding method $(4,5)$. The invarlant embedding method is applicable to one-dimensional problems; whereas, the response matrix method can treat one-, two-, or three-dimensional problems. In the response matrix method, the reactor is assumed to be divided into a collection of contiguous cells. Response matrix elements are computed for each different type of cell to describe the neutron current leaving the cell due to a given current

* Work done under USAEC Contract No. AT (07-2)-1. 
entering the cell. A criticality equation formulated by conserving neutrons at each cell interface in the reactor is written as

$$
\lambda \vec{J}=R \vec{J}
$$

where $\lambda$ is a current eigenvalue, $R$ is a composite response matrix formed from individual cell response matrix elements, and $\mathrm{J}$ is a vector describing interface cell currents throughout the reactor.

In this paper, we w111 discuss recent developments on three aspects of this equation: the calculation of the static response matrix, the timedependent problem, and the solution of the criticality Eq. (1). A discussion of future developments is also provided.

\section{STATIC RESPONSE MATRIX}

The objectlve is to derive the cell response matrix, which is defined by the following equation:

$$
\mathrm{J}^{\mathrm{OUT}}=\mathrm{RJ}^{\mathrm{IN}}
$$

where JOUT is the outgoing cell current due to an incoming cell current $\mathrm{J}^{\mathrm{IN}}$. The cell is assumed to be in a vacuum and can be of any shape; square or hexagonal cells are applicable to most reactor designs. In our discussions, we shall consider hexagonal cells and limtt the initial 1llustrations to twodimensional geometry. Figure 1 lllustrates the process described by Eq. (2).

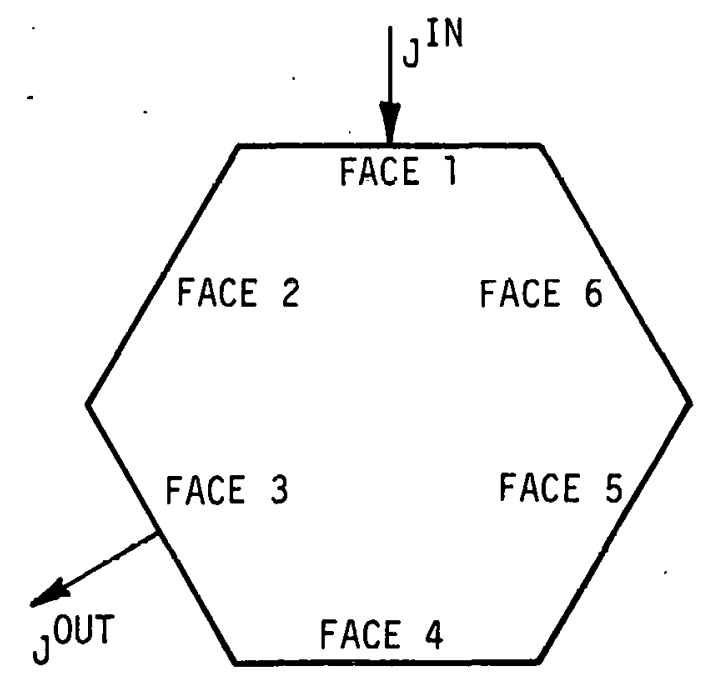

FIGURE 1. Cell Response Matrix Element $R(1 \rightarrow 3)$ for TwoDimensional Hexagonal Geometry 
We shall derive the response matrix using the collision probability form of the transport equation, although other theorles are equally useful. We assume that the cell is divided into reglons in which the flux is constant. For simplicity, we will also assume isotropic sources. With these approximations, the transport equation is written as

$$
\Sigma(n) \phi(n)=\sum_{m} P^{V V}(n, m) S(m)+\sum_{k} P^{V S}(n, k) J^{I N}(k)
$$

where the first flight collision probabilities $\mathrm{pWV}$ and $\mathrm{p}^{\mathrm{VS}}$ are defined in the usual manner $(\underline{6}), \phi(n)$ is the flux in region $(n), \Sigma(n)$ is the total cross section in region $(n), J^{I N}(k)$ is the incoming current on face (k) of the cell, and $S(m)$ is the source in region $(m)$. For brevity, Eq. (3) is divided by $\Sigma(n)$ and expressed in vector form

$$
\phi=T^{V V_{S}}+T^{V S}{ }_{J}^{I N}
$$

where the volume transport elements $\mathrm{T}^{\mathrm{VV}}$ and $\mathrm{T}^{\mathrm{VS}}$ are $\mathrm{T}^{\mathrm{VV}}=\Sigma^{-1} \mathrm{P} V$ and $\Sigma^{-1} \mathrm{P} V S$.

The source is given by

$$
s=\Sigma_{s} \phi+\frac{1}{k} v X \Sigma_{f} \phi
$$

where the first and second terms represent scattering and fission sources, respectively, and $\mathrm{k}$ is a multiplication constant. We write the cell outgoing current as

$$
\mathrm{J}^{\text {OUT }}=\mathrm{T}^{S V_{S}}+\mathrm{T}^{\mathrm{SS}} \mathrm{JN}
$$

where $\mathrm{T}^{\mathrm{SV}}$ and $\mathrm{T}^{\mathrm{SS}}$ are the surface transport elements. Comblning Eqs. (4), (5), and (6) leads to the result

$$
\mathrm{J}^{\text {OUT }}=\left\{\mathrm{T}_{\mathrm{G}}\left[\mathrm{I}-\mathrm{T}_{\mathrm{G}}\right]^{-1} \mathrm{~T}^{\mathrm{VS}}+\mathrm{T}^{S S}\right\} \mathrm{J}^{\mathrm{IN}}
$$

where the matrix

$$
G=\Sigma_{s}+\frac{1}{k} \nu \times \Sigma_{f}
$$

Comparing Eqs. (2) and (7) shows that the response matrix is

$$
R=T^{S V}\left[I-T^{V} V_{G}\right]^{-1}{ }_{T} V S+T^{S S}
$$

$$
\text { S3 - L95 - p3 }
$$


In practice, Eq. (8) is not used to evaluate the response matrix because the cost of inverting the matrix in this equation is of ten too expensive. Instead, Eqs. (4), (5), and (6) are solved by iterative methods for various elements of the response matrix through appropriate initialization of the fixed incoming current vector $\mathrm{J}^{\mathrm{IN}}$. By setting all but one of the elements of the vector JIN to zero and solving these equations, a column of the response matrix is determined. Repeating the process with a new non-zero element will enable determination of the remaining columns. These calculations are not too costly if one can take into account any cell symmetry. Figure 2 illustrates the response matrix for a hexagonal cell containing only $\mathrm{D}_{2} \mathrm{O}$ (sixfold symmetry). The matrix was generated for one neutron speed. This response matrix has 36 elements, of which only 4 are unique. All 4 unique numbers are in each column and therefore are determined in a single calculation. This observation saves both computer time and storage. If the cell geometry were not sixfold symmetric, then all 36 elements would most likely be different, and 6 calculations would be required to evaluate the matrix.

$$
\begin{aligned}
& \text { Incident Faces } \rightarrow \\
& \left(\begin{array}{cccccc}
\mathrm{A} & \mathrm{B} & \mathrm{C} & \mathrm{D} & \mathrm{C} & \mathrm{B} \\
\mathrm{B} & \mathrm{A} & \mathrm{B} & \mathrm{C} & \mathrm{D} & \mathrm{C} \\
\stackrel{\tilde{D}}{ \pm} \\
\stackrel{+}{x} \\
+ \\
+
\end{array}\right. \\
& A=0.473 \quad B=0.192 \quad C=0.0518 \quad D=0.0382
\end{aligned}
$$

FIGURE 2 Response Matrix for Hexagonal Cell Containing Only Heavy Water

The response matrix as given in Eq. (8) is an implicit function of the multiplication factor $\mathrm{k}$, which is not usually known until the reactor problem is solved. This problem can be handled in two ways: computing the response matrices for two or more values of $k$ and interpolating while solving the criticality equation; or separating the fission contributions from the cell response. At the Savannah. River Laboratory, we have chosen the second method. The response matrix is written a's 


$$
R(k)=R_{D}+R_{I}(k)
$$

where $R_{D}$ is the direct component and represents those neutrons that enter the cell and suffer at most only scattering collisions before exiting the cell. The matrix $\mathrm{RI}_{\mathrm{I}}$ is the indirect component and represents those neutrons exiting the cell resulting from fissions. The direct component $R_{D}$ is given by

$$
R_{D}=T^{S V_{\Sigma}}\left[I-T^{V V_{S}}\right]^{-1} T^{V S}+T^{S S}
$$

The indirect component is written as

$$
R_{I}(k)=\frac{1}{k} R_{I}^{(1)}+\frac{1_{1}}{k^{2}} R_{I}^{(2)}+\cdots
$$

where the matrices $\mathrm{R}_{I}^{(1)}$ are independent of the parameter $k$ and are given by

$$
R^{(i)}=T^{S V}\left[\Sigma_{s} \phi^{(i)}+\nu \times \Sigma_{f} \phi^{(i-1)}\right] \quad i>0
$$

where

$$
\phi^{(i)}=\left\{\left[I-T^{V V_{S}}\right]^{-1} v X \Sigma_{f}\right\}^{I}\left[I-T^{V V}\right]^{-1} T^{V S} \quad 1 \geqslant 0
$$

The response $R_{I}^{(i)}$ and the flux $\phi^{(i)}$ represent the contributinns from the (i-1) generation of fission neutrons. In practice, these matrices are computed by following each generation of fission neutrons separately. Fission neutron sources from one generation are used as the source for the next, and so on. We have found that this series converges quite rapidly for all cell types studied, and only a few terms need be computed and retained. Eq. (13) may be used to sum the remaining terms:

$$
\lim _{i \rightarrow \infty} \frac{\left[R^{(i+1)}\right]}{\left[R^{(i)}\right] j k \ell \ldots}=k_{v} \cdots \quad \quad 1>0
$$

where $\left[R^{(i)}\right]_{j k \ell} \ldots$ is an element of the response matrix for generation ( $\left.i\right)$, and $\mathrm{kv}_{\mathrm{v}}$ is the effective multiplication constant for the cell in a vacuum.

$$
\text { S3 - L95 - p5 }
$$


If a cell contains no fissionable material, then

$$
R^{(1)}=0
$$

and the response matrix is simply the direct response matrix.

Response matrices are computed for each cell type in the reactor, and the composite response matrix used in the criticality Eq. (1) is formed. The criticality equation is usually solved by adjusting the $k$ parameter until the eigenvalue $\lambda$ is 1 . The parameter $k$ computed in this manner is the effective multiplication constant for the reactor.

The cell currents determined by solving the criticality equations are themselves of minimal importance; however, they can be used to compute cell fluxes and reaction rates. Various other response matrices can be computed and stored at the same time the current-response matrices are computed. The flux response matrix

$$
R_{F}=\left[I-T^{V V}\right]^{-1} T^{V S}
$$

can then be used to evaluate the cell flux

$$
\phi=R_{R} J^{I N}
$$

where $\mathrm{J}^{\mathrm{IN}}$ is the cell current edited from the solution of Eq. (1).

Varlous reaction response functions can similarly be defined as

$$
R_{R}=\Sigma_{R}\left[I-T^{V V_{G}}\right]_{T}^{-1}{ }_{T}^{V S}
$$

for reaction type $R$. Fission, capture, and scattering reaction response matrices can be computed, and Eq. (12) can be used to express these matrices as explicit functions of the multiplication constant $\mathrm{k}$.

The response matrices as discussed above have been calculated using $S_{n}$ methods (2) , diffusion theory $(\underline{4,5})$, and Monte Carlo techniques (1) as wel1 as integral transport theory $(\underline{1})$. Considerations of speed and accuracy dictate the appropriate method. Splitting the response matrix into direct and indirect components provides the possibility of mixing methods in the calculation of the total matrix, for example, using Monte Carlo to compute the direct contribution and integral transport to compute the indirect contribution. This approach has been successful in minimizing lateral flux spreading effects. 


\section{Lateral Flux Spreading}

The lateral flux spreading effect (7) represents a significant source of error in the computation of the response matrix. This error arises because of the numerical treatment of the spatial flux distribution in the cell, that is, the assumption of a flat flux in a cell region. Neutrons colliding in a region become sources spread uniformly over the entire region. The net effect is that the transmission of neutrons from the entrance face to an exit face is overestimated, and leakage computed in reactor calculation is too large. The error is minimized by increasing the number of regions in the cell; however, this also increases the response matrix computation time. The effect of number of regions on the response elements is illustrated in Figure 3. Most of the flux spreading error occurs in the calculation of the direct response matrix. Using a more precise calculation here, such as Monte Carlo, will nearly eliminate this error.

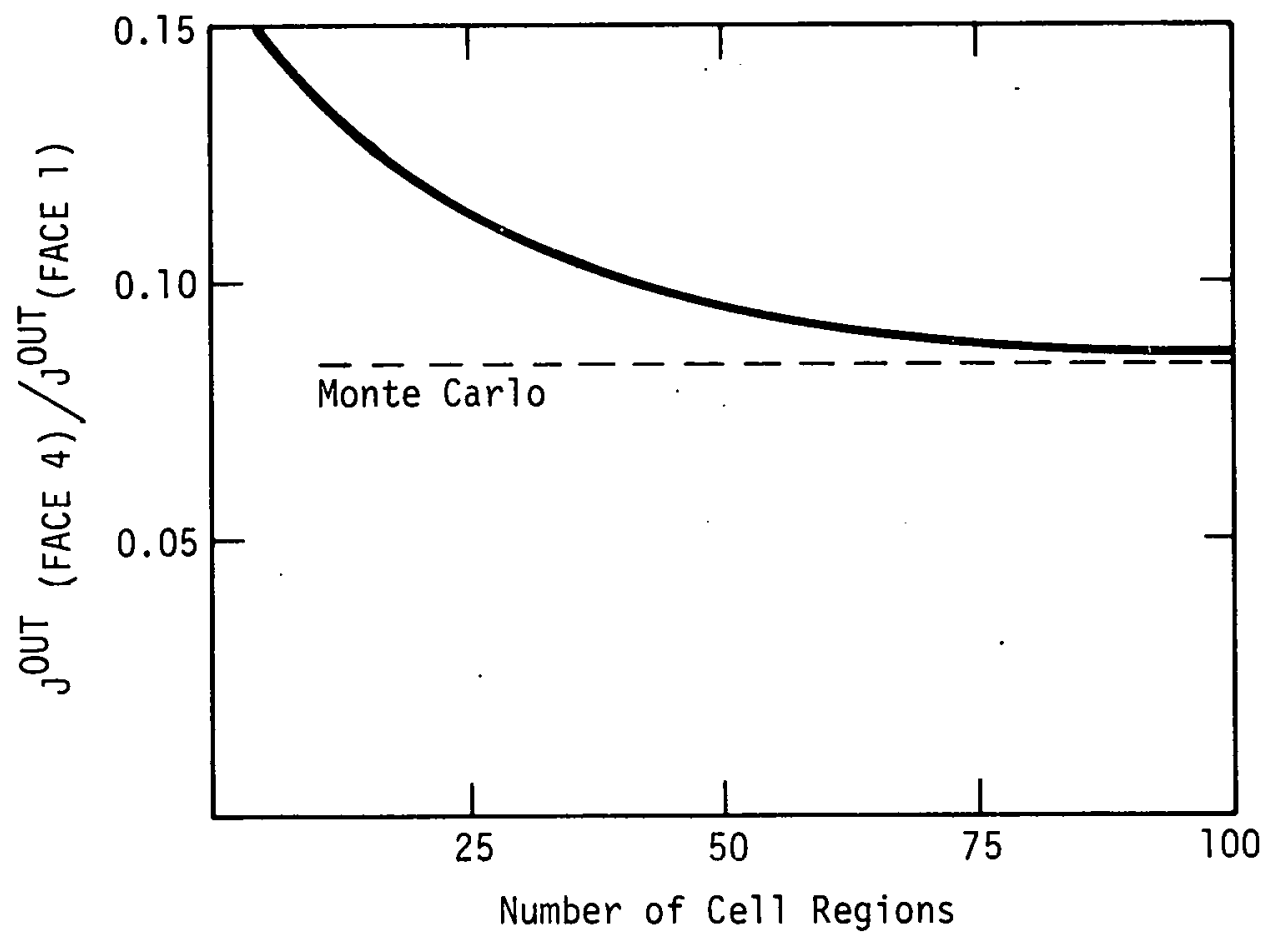

FIGURE 3 Ratio of the Outgoing Current of Faces 4 and 1 for Neutrons Entering Face 1 as a Function of the Number of Regions in the Cell

The cell is described in Figures 1 and 2. 


\section{Choice of Current Modes}

The general form of the time-independent response matrix is

$$
R\left(E \rightarrow E^{\prime}, \vec{X} \rightarrow \vec{X}^{\prime}, \vec{\Omega} \rightarrow \vec{\Omega}^{\prime}\right)
$$

where the unprimed/primed variables indicate energy, position, and direction of the incoming/outgoing cell currents. This form of the matrix is quite general and leads to good results if enough resolution in the variable components are retained in defining response matrices used in any given calculation. Computationally, this form of the matrix is usually simplified by making several approximations. Averaging within energy groups leads to the form

$$
R\left(G \rightarrow G^{\prime}, \vec{X} \rightarrow \vec{X}^{\prime}, \overrightarrow{\Omega^{\prime}} \rightarrow \vec{\Omega}^{\prime}\right)
$$

where $G$ is the group number. Experience in heavy water lattices has shown that 2 to 6 groups are sufficient to treat any spectral effects. Numbering the sides of the cell and defining a set of current distribution functions lead to the form

$$
R\left(G \rightarrow G^{\prime}, n \rightarrow n^{\prime}, m \rightarrow m^{\prime}\right)
$$

where $\mathrm{n}$ is a cell face number, and $\mathrm{m}$ refers to a particular distribution function describing currents incident on or emerging froin a cell face.

Recently, oome effort has been directed to determining the effect of different angular and spatial distribution functions in heavy water lattices on the prediction of important reactor properties ( 8 ). The results of the study indicate that for many reactor problems, a spatially flat, cosine currents approximation describing the interface currents. gives good results and leads to relatively simple response matrix elements of the form

$$
R\left(G \rightarrow G^{\prime}, n \rightarrow n^{\prime}\right)
$$

There are some cases (for example voiding), however, where more spatial and angular resolution of the neutron current at a cell interface is required. For these cases, linear spatial and "double-cosine" angular distributions give excellent results $(\underline{8})$. 


\section{Multidimensional Problems}

To date, only two-dimensional codes in square and hexagonal geometry have been reported in the literature. In most computer codes, the third dimension is treated using appropriate $\mathrm{DB}^{2}$ contributions added to the absorption cross section at the time the cell response matrix is computed. A novel approach used in the code RASCAL ( 1 ) uses response matrices computed for infinitely long cells and corrects the matrix elements for axial leakage by scaling total outgoing cell leakage (radial and axial) and cell flux to conserve the total neutron population in each cell. This approach appears to work well for heavy water lattices.

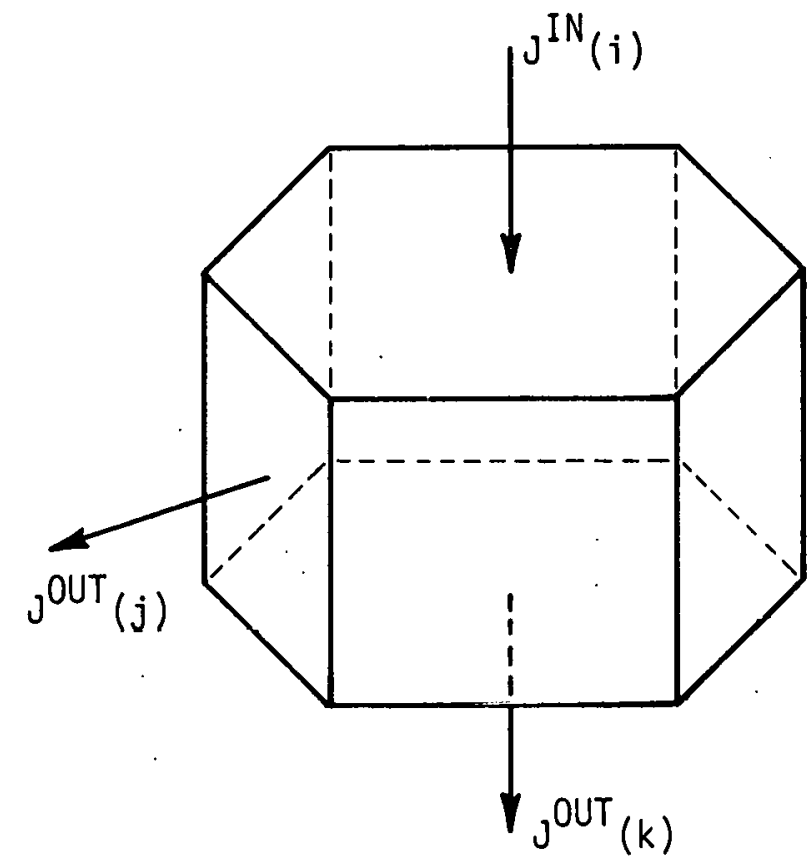

FIGURE 4 Cell Response Matrix Elements in Three-Dimensional Hexagonal Geometry

A general three-dimensional treatment will require computing response matrices of the type depicted in Figure 4. Such calculations appear to be expensive today (using multigroup transport theory) from both a storage and computational point of view; however, new approximate methods or the next generation of computers might handle this problem. 


\section{TIME-DEPENDENT PROBLEM}

The time-dependent response equation is expressed as

$$
J^{\text {OUT }}(t)=\int_{-\infty}^{t} R\left(t, t^{\prime}\right) J^{I N}\left(t^{\prime}\right) d t^{\prime}
$$

where $R\left(t, t^{\prime}\right)$ is the time-dependent response function and represents the outgoing cell currents at time $t$ due to a burst of incoming neutrons at time $t$ '. In this form, the response matrix has three components each having significantly different time constants. The fastest component results from the transmission of uncollided neutrons, next fastest results from scattered and prompt fission neutrons, and the slowest component results from the escape of delayed neutrons. Reference 3 solves this equation for slab geometry and evaluates the response matrix from the solution of the time-dependent double-Po equations. The three components of the response matrix are approximated, and a forward integration technique is used to evaluate the integral in Eq. (21). The currents are linearly interpolated between time $t_{n}$ and $t_{n-1}$ by

$$
J^{I N}(t)=J_{n}^{I N}-\frac{J_{n}^{I N}-J_{n-1}^{I N}}{\Delta t_{n}}\left(t_{n}-t\right) .
$$

where $J_{n}^{I N}=J^{I N}\left(t_{n}\right)$.

Substituting (22) into (21) results in the system of equations

$$
J_{n}^{\text {OUT }}=\left(\gamma_{0}^{n}-\gamma_{1}^{n}\right) J_{n}^{T N}+\gamma_{1}^{n} J_{n-1}^{I N}+f_{n}
$$

where $\gamma_{0}^{\mathrm{n}}$ and $\gamma_{1}^{\mathrm{n}}$ are moments defined by

$$
\gamma_{i}^{n}=\frac{1}{\left(\Delta t_{n}\right)^{i}} \int_{0}^{\Delta t_{n}^{\prime}} R\left(t_{n}, t_{n}-\tau\right) \tau^{i} d \tau
$$

and $f_{\mathfrak{n}}$ is given by

$$
f_{n}=\int_{t_{n-2}}^{t} R\left(t_{n}, t^{\prime}\right) J^{I N}\left(t^{\prime}\right) d t^{\prime}+\int_{-\infty}^{t} n-2
$$

Equation (25) is approximated by assuming only delayed neutrons contribute to the second integral, which is a good approximation if $\Delta t_{n-1}$ is large compared to the prompt response time. If this is true, then 


$$
R\left(t_{n}, t^{\prime}\right) \approx e^{-\lambda \Delta t_{n}} R\left(t_{n-1}, t^{\prime}\right) \quad t^{\prime}<t_{n-2}
$$

and

$$
f_{n}=\int_{t_{n-2}}^{t} R\left(t_{n}, t^{\prime}\right) J^{I N}\left(t^{\prime}\right) d t^{\prime}+e^{-\lambda \Delta T} n f_{n-1}
$$

Reference 3 shows that this method provides good results if care is taken in the evaluation of the moments $\gamma_{0}$ and $\gamma_{1}$.

An alternative approach being considered to treat the time-dependent problem is to separate the prompt and delayed responses. The time-dependent response equations are then written as

$$
J_{(t)}^{\text {OUT }}=\int_{-\infty}^{t} R_{P}\left(t, t^{\prime}\right) J^{I N}\left(t^{\prime}\right) d t^{\prime}+\int_{-\infty}^{t} R_{S}\left(t, t^{\prime}\right) \sum_{1} \lambda_{1} c_{1}\left(t^{\prime}\right)
$$

where the delayed neutron precursors are governed by

$$
\frac{d C_{1}}{d t}=-\lambda_{1} C_{1}+\beta_{1} \nu \Sigma_{f} \phi(t)
$$

In this model the prompt $R_{p}$ and delayed $R_{S}$ response matrices look very much like prompt responses and are characteristic only of cell flight times. The emission of delayed neutrons is determined by the explicit treatment of the prenirsors. In order to save cumputer time, it would be advantageous if these matrices can be expressed in terms of the static response matrix elements discussed earlier. This is posstble by separating the time and space dependence. An obvious approximation is

$$
R_{p / s}\left(t, t^{\prime}\right)=R_{p / s} f_{p / s}\left(t, t^{\prime}\right)
$$

where

$$
\int_{-\infty}^{t} f\left(t, t^{\prime}\right) d t^{\prime}=1
$$

$$
\text { S3 - L95 - p11 }
$$


and where the matrices $R_{p} / s$ are static response matrices. We approximate the: static response matrices by

$$
R_{p}=R_{D}+R_{I}\left(\frac{1}{I-\beta}\right)
$$

and

$$
R_{s}=\frac{R_{I} J^{I N}}{\sum_{i j k \ldots}\left(R_{f}{ }^{I N}\right)_{i j k \ldots} . .}
$$

where $\beta=\sum_{i} \beta_{i}, R_{I}$ and $R_{D}$ are static response matrices derived earlier, and $R_{f}$ is the static reaction response matrix corresponding to the cell fisston rate. The production rate in Eq. (29) may be approximated by the expression

$$
v \Sigma_{\mathrm{f}} \phi(t)=\mathrm{R}_{\nu f^{\mathrm{J}}} \mathrm{J}^{\mathrm{IN}}(\mathrm{t})
$$

where $R_{\nu f}$ is a static reaction response matrix corresponding to $\nu$-fission reactions.

The results of using this model will be reported later.

\section{SOLUTION OF THE CRITICALITY EQUATION}

The matrix in Eq. (1) is essentially different in its properties from the matrices dealing directly with the neutron flux such as diffusion theory. The latter usually is characterized by a three-point finite difference scheme for which many well-established solution methods exist. Only one "established solution method" appears to exist for the response matrix equation. Our experience in solving this equation for two-dimensional multigroup problems indicates that the standard power iteration method works acceptably well. At least one author has tried to reformulate the response equations in order to use standard acceleration techniques. Reference 9 shows that in one-dimension, Eq. (1) can be cast in a three-point form, for which many standard acceleration techniques apply. It is not clear however that this procedure is applicable for twoor three-dimensional problems.

A coarse-mesh technique employed in the RASCAI code saves significant time if many modes are used to define the interface currents. The code periodically in the fiteration will collapse the mulit-mode representation to a singlemode representation and solve the criticality equation for the reduced problem. The single-mode solution is then used to scale the intermediate multi-mode solution. 


\section{FUTURE DEVELOPMENTS}

It is clear that much work must be done in developing acceleration techniques for solving the reactor criticality equation. Also, improved methods must be developed to treat three-dimensional problems. Of real importance today are the methods to reduce the computational time required to evaluate the two-dimensional response matrix. Integral transport approaches are favored because they are accurate and usually are better suited to treat irregular geometric lattice patterns; however, the computation time to generate the response matrix using these methods increases as the square of the number of regions in the cell. The need to have sufficient regions to overcome lateral flux spreading errors can result in costly calculations. A new integral treatment whose computation time has a linear dependence on the number of regions is the "transmission currents technique" (10). This method is fast and looks ideal for computing both two- and three-dimensional response matrices.

The development of time-dependent methods will continue. This effort should be directed at utilizing the static response matrices.

\section{REFERENCES}

1. R. J. Pryor and W. E. Graves, "Response Matrix Method for Treating Reactor Calculations," Proc. Mathematical Models and Computational Techniques for Analysis of Nuclear Systems, Apri1 9-11, 1973, Ann Arbor, Mich. USAEC Report CONF-730414-P2, Technical Information Center, Oak Ridge, Tenn., p VII-179 (1973).

2. H. S. Bailey, "Response Matrix Analysis for Fast Reactors," Proc. Mathematical Models and Computational Techniques for Analysis of Nuclear Systems, Apri1 9-11, 1973, Ann Arbor, Mich. USAEC Report CONF-730414-P2, Technical Information Center, Oak Ridge, Tenn., p VII-187 (1973).

3. J. M. Sicillail àl A. Leonard, Trans. Amer. Nucl. Soc. 1\%, 252 (1973).

4. A. Shimizu and K. Aoki, Application of Invariant Embedding to Reactor Physics, Academic Press, New York (1972).

5. K. Aok1 and A. Shimizu, J. Nucz. Sci. Technol. 2, 149 (1965).

6. D. Emendörfer, "Physics Assumptions and Applications of Collision Probability Methods," Proc. Mathematical Models and Computational Techniques for Analysis of Nuclear Systems, April 9-11, 1973, Ann Arbor, Mich. USAEC Report CONF-730414-P2, Technical Information Center, Oak Ridge, Tenn., p VII-136 (1973).

7. W. L. Filippone, Nucl. Sci. Eng. 52, 23 (1973).

8. R. J. Pryor, Trans. Amer. Nucl. Soc. 17, 262 (1973).

9. Z. Weiss, Nucl. Sci. Eng. 48, 235 (1972).

10. M. M. Anderson and H. C. Honeck, "An Interface Current Technique for TwoDimensional Cell Calculations," Proc. Mathematical Models and Computational Techniques for Analysis of Nuclear Systems, April 9-11, 1973, Ann Arbor, Mich. USAEC Report CONF-730414-P2, Technical Information Center, Oak Ridge, Tenn., p I-53 (1973). 